\title{
Feigenblatt oder fiskalische Zeitenwende? Zur potenziellen Wirksamkeit des Fiskalvertrags
}

\author{
Friedrich Heinemann, Marc-Daniel Moessinger und Steffen Osterloh*
}

Die europäische Schuldenkrise hat die Schwächen des fiskalischen Regelwerks in der Europäischen Wirtschafts- und Währungsunion umfassend aufgedeckt. Spätestens seit Zuspitzung der Vertrauenskrise im Frühjahr 2010 ist nicht mehr bestreitbar, dass das bis dato existierende und durch den Stabilitäts- und Wachstumspakt (SWP) definierte Verfahren bei übermäßigen Defiziten ${ }^{1}$ über keine ausreichende Wirksamkeit verfügt und auch die sogenannte No-Bailout-Klausel des Art. 125 AEUV nicht über eine ausreichende Glaubwürdigkeit zur Herstellung von Disziplinierungsdruck verfügt hat.

Die akute Krise mit ihren schwerwiegenden Erschütterungen hat in kurzer Zeit viele bis dahin bestehende Tabus beseitigt. Die Euro-Staaten haben bedeutsame Beistandskreditfazilitäten geschaffen, die im Konflikt mit der Intention der No-Bailout-Klausel stehen. Diese waren zunächst temporär konstruiert (Europäische Finanzstabilisierungsfazilität und -mechanismus, EFSF und EFSM), sollen aber bereits im Jahr 2012 durch einen permanenten Beistandsmechanismus, den Europäischen Stabilitätsmechanismus (ESM), ersetzt werden. Die Europäische Zentralbank hat sich mit ihren Interventionen - Anleihekäufe und sehr langfristige Geldmarktgeschäfte - stark in die Politik zur Eindämmung der Krise einbinden lassen und dabei Grundprinzipien der Maastrichter Währungsverfassung wie etwa das Verbot von Zentralbankkrediten an die Mitgliedstaaten de facto temporär verletzt. Neue europäische Vereinbarungen haben seitdem nicht nur im Bereich der fiskalischen Governance umfangreiche Innovationen hervorgebracht. Auf makroökonomischem Gebiet soll der EuroPlus-Pakt ${ }^{2}$ auf eine stärkere Koordinierung der Wirtschaftspolitik und das Verfahren bei einem makroökonomischen Ungleichgewicht auf eine frühzeitige Bekämpfung gesamtwirtschaftlicher Ungleichgewichte hinwirken. Hinzu treten neue Verfahren der Politikkoordination wie die Abstimmung der nationalen Haushaltsplanung im Rahmen des Europäischen Semesters. ${ }^{3}$

Im Mittelpunkt dieses Beitrags stehen demgegenüber die Innovationen im Hinblick auf ein stärkeres fiskalisches Regelwerk. Auf dem haushaltspolitischen Feld ist es nicht nur zu einer graduellen Fortentwicklung durch die Verschärfung des SWP, sondern qualitativ zu einem gänzlichen Neuansatz gekommen. Während der SWP europarechtliche Regeln und

* PD Dr. Friedrich Heinemann, Leiter des Forschungsbereichs Unternehmensbesteuerung und Öffentliche Finanzwirtschaft, Zentrum für Europäische Wirtschaftsforschung, Mannheim.

Marc-Daniel Moessinger, Wissenschaftlicher Mitarbeiter im Forschungsbereich Unternehmensbesteuerung und Öffentliche Finanzwirtschaft, Zentrum für Europäische Wirtschaftsforschung, Mannheim.

Dr. Steffen Osterloh, Wissenschaftlicher Referent für Europapolitik beim Sachverständigenrat zur Begutachtung der gesamtwirtschaftlichen Entwicklung, Wiesbaden.

Dieser Beitrag spiegelt die persönliche Meinung der Autoren wider. Diese entspricht nicht notwendigerweise der Auffassung des Sachverständigenrates zur Begutachtung der gesamtwirtschaftlichen Entwicklung.

1 Art. 126 Vertrag über die Arbeitsweise der Europäischen Union (AEUV) i.V.m. Protokoll (Nr. 12) über das Verfahren bei einem übermäßigen Defizit, in: Amtsblatt der EU, Nr. C 83 vom 30. März 2012, S. 279-280.

2 Vgl. Daniel Gros/Cinzia Alcidi: Was bringt der „Euro-plus-Pakt“?, in: integration 2/2011, S. 164-171.

3 Für einen Überblick über die Schlüsselentscheidungen seit Ausbruch der Krise siehe Tobias Kunstein/Wolfgang Wessels: Die Europäische Union in der Währungskrise: Eckdaten und Schlüsselentscheidungen, in: integration 4/2011, S. 308-322. 
Sanktionsmöglichkeiten definiert, zielen weitere Innovationen nun auf eine stärkere Indienstnahme harmonisierter Fiskalregeln und haushaltspolitischer Prozesse auf der nationalen Ebene ab. Dies gilt bereits für die Richtlinie über die Anforderungen an die haushaltspolitischen Rahmen der Mitgliedstaaten ${ }^{4}$ und noch mehr für den am 2. März 2012 unterzeichneten Vertrag über Stabilität, Koordinierung und Steuerung in der Wirtschafts- und Währungsunion (Fiskalvertrag). ${ }^{5}$

Obwohl die Richtlinie und der Fiskalvertrag von unterschiedlicher rechtlicher Qualität sind (Rechtsetzung durch die Europäische Union versus zwischenstaatlicher Vertrag), haben sie eine analoge Zielrichtung: Beide setzen darauf, Vorgaben für den nationalen Gesetzgeber zur Anpassung seines nationalen Regelwerks zu machen. Hier sollen somit das nationale Haushaltsrecht, und damit nationale Aufsichtsbehörden und Gerichte, stärker als bisher in Dienst genommen werden, um eine nachhaltige Budgetpolitik zu erzwingen. Diese Entwicklung ist auch integrationspolitisch bedeutsam. Während die Regeln des SWP bislang die große nationale Varianz von haushaltspolitischen Institutionen respektierten, greifen die neuen Vereinbarungen in die nationalen Institutionen ein und sollen ein bestimmtes Maß an Harmonisierung von Haushaltsregeln erzwingen.

Ziel dieses Beitrags ist es, die Substanz dieser Integrations- und Harmonisierungsschritte im Bereich der Haushaltspolitik zu bewerten. Hierzu sind durchaus Erfahrungen aus dem nationalen und subnationalen Bereich (Schweizer Kantone, US-Bundesstaaten) verfügbar. Fiskalregeln, wie sie nun durch den Fiskalvertrag für die teilnehmenden Staaten verpflichtend werden sollen, können daher im Lichte dieser Vorläufer beurteilt werden.

Im nächsten Abschnitt werden zunächst die erfolgten Korrekturen am SWP und die Innovationen im Bereich der nationalen haushaltspolitischen Regeln vorgestellt und voneinander abgegrenzt. Daran schließt sich ein Überblick über die Literatur zur Wirksamkeit von nationalen und subnationalen Fiskalregeln an. Auf dieser Basis kann dann eine Einschätzung erfolgen, inwieweit der Fiskalvertrag tatsächlich einen Beitrag zu mehr fiskalischer Stabilität leisten kann.

\section{Innovationen der fiskalischen Governance}

\section{Funktion neuer fiskalischer Regeln in der Krisenbewältigungs-Strategie}

Im Bemühen um die Eindämmung der Vertrauenskrise hinsichtlich der europäischen Staatsverschuldung standen von Beginn an Versuche zur Verbesserung des fiskalischen Regelwerks im Fokus. Die grundsätzliche Sinnhaftigkeit dieses Ansatzes steht außer Frage. Eine tiefer liegende Ursache der Schuldenkrise ist die kurzfristige Attraktivität schuldenfinanzierter Staatsausgaben im politischen Wettbewerb. Eine ursachenadäquate Strategie der Krisenbekämpfung sollte an dieser Verschuldungsneigung ansetzen und versuchen, institutionelle Vorkehrungen zu treffen, welche einer permanenten Akkumulation immer neuer öffentlicher Schulden entgegenwirken.

Neue fiskalische Regeln sind auf mittel- und langfristige Wirkungen ausgelegt und kein unmittelbarer Beitrag zur Eindämmung der akuten Krisensituation. Gleichwohl können sie, wenn sie glaubwürdig ausgestaltet sind, bereits durch ihre Beeinflussung der Erwartungs-

4 Richtlinie 2011/85/EU des Rates über die Anforderungen an die haushaltspolitischen Rahmen der Mitgliedstaaten, in: Amtsblatt der EU, Nr. L 306 vom 23. November 2011, S. 41-47.

5 Abgedruckt in: Gesetzentwurf der Fraktionen der CDU/CSU und FDP. Entwurf eines Gesetzes zu dem Vertrag vom 2. März 2012 über Stabilität, Koordinierung und Steuerung in der Wirtschafts- und Währungsunion, Bundestagsdrucksache 17/9046. 
bildung an den Märkten für Staatsanleihen eine Entlastung bewirken. Wenn überzeugende neue Regeln für die Zukunft eine Trendwende im Verschuldungspfad eines Staates signalisieren, dann kann dieses Signal unmittelbar zu einer verbesserten Bonitätseinschätzung und damit zu fallenden Renditen führen. Die Regelungen zielen somit darauf ab, kurzfristig das Vertrauen der Finanzmärkte in die Haushaltspolitik auf nationalstaatlicher Ebene wiederherzustellen und langfristig solide Staatsfinanzen sicherzustellen.

Vor diesem Hintergrund basiert die Kritik, solche Fiskalregeln seien blindwütige Austerität und würden die Krise noch verschärfen, auf einem grundlegenden Missverständnis. ${ }^{6}$ Fiskalregeln, wie sie im Fiskalvertrag vereinbart wurden, lassen der Budgetpolitik Spielraum, um im Konjunkturverlauf ,zu atmen'. Sie verpflichten aber dazu, in konjunkturellen Normalzeiten (und noch mehr in überdurchschnittlich guten Wachstumsjahren) Sparsamkeit an den Tag zu legen. In der akuten gegenwärtigen Schuldenkrise, in der manche Staaten überhaupt keine Chance haben, ihr Budget kurzfristig auch nur in die Nähe der Nulllinie zu bringen, signalisiert die Etablierung solcher Regeln den Kreditgebern, dass diese Staaten in der Zukunft unter besseren Bedingungen ihre Verschuldung eindämmen werden, auch wenn die aktuellen Budgetzahlen dies noch nicht zeigen können. Ein Verzicht auf glaubwürdige Fiskalregeln würde erst recht kompromisslose Sparmaßnahmen in der Gegenwart erzwingen, weil dann letztlich nur eine Verbesserung der Ist-Zahlen bliebe, um Kreditgebern eine Wende in der Schuldenpolitik zu signalisieren.

Um durch neue Regelungen neues Vertrauen zu schaffen, wurden im Krisenverlauf zwei fundamental unterschiedliche Ansatzpunkte gewählt. Zunächst stand eine Stärkung der auf der supranationalen Ebene bereits bestehenden europäischen Haushaltsregeln des SWP im Vordergrund. Daran anschließend verlagerte sich die Reformpolitik darauf, in einem koordinierten Verfahren neue nationale Fiskalregeln zu etablieren.

\section{Die Reform des Stabilitäts- und Wachstumspakts}

Das Scheitern des SWP in der Zeit vor der akuten Verschuldungskrise hatte ganz offenkundig seine Ursachen in dessen mangelnder Durchsetzbarkeit, die sich in einer Nichtbestrafung selbst derjenigen Staaten gezeigt hat, welche regelmäßig die im Pakt vorgegebenen Grenzwerte überschritten haben. Dabei hatte der sogenannte korrektive Arm des SWP, in dessen Rahmen die Neuverschuldung auf 3 Prozent des Bruttoinlandsprodukts (BIP) begrenzt wurde, bereits über einen - zumindest auf dem Papier - durchaus bedeutsamen Sanktionsmechanismus verfügt. Dieser sah bei einem Verstoß ein Verfahren unter Mitwirkung von Europäischer Kommission und Rat der Europäischen Union vor, an dessen Ende Sanktionen bis hin zu Geldstrafen drohten. ${ }^{7}$ Flankiert wurde dieses vom sogenannten präventiven Arm, der die langfristige Nachhaltigkeit der Haushaltspolitik sicherstellen sollte, jedoch nicht sanktionsbewehrt war.

Die konsequente Umsetzung dieses bestehenden Instrumentariums hätte die verantwortungslose Hauspolitik in einigen Staaten, etwa Griechenland, durchaus verhindern können. Die Regelungen des SWP waren jedoch spätestens seit den im Jahr 2005 von Deutschland und Frankreich vorangetriebenen Aufweichungen praktisch sämtlicher Automatismen be-

6 Friedrich Heinemann/Florian Misch/Marc-Daniel Moessinger/Christoph Schröder/Mustafa Yeter: Stellungnahme zum Gesetzespaket Fiskalvertrag, ESM und Bundesschuldenwesen, Anhörung Deutscher Bundestag, Haushaltsausschuss, 7. Mai 2012, Mannheim.

7 Verfahren bei einem übermäßigen Defizit. 
raubt. ${ }^{8}$ Insbesondere erwies es sich als fatal, dass in allen Schritten des Defizitverfahrens eine qualifizierte Mehrheit des Rates der Europäischen Union erforderlich war. Diese kam jedoch selten zusammen, da viele Mitgliedstaaten selbst einem Verfahren unterlagen oder dieses fürchten mussten, sodass ,(potenzielle) Sünder über Sünder ${ }^{6}$ zu richten hatten. Aus diesem Grund wurden die möglichen Sanktionen nie angewendet, sodass der SWP letztlich jegliche abschreckende Wirkung eingebüßt hatte. Als weiteres Problem ist rückwirkend zu erkennen, dass der SWP vor allem auf die Begrenzung der Neuverschuldung ausgerichtet war, wohingegen das Schuldenstandskriterium (Begrenzung auf 60 Prozent des BIP) trotz regelmäßiger Überschreitung in einer Vielzahl an Ländern nicht berücksichtigt wurde.

Trotz dieser geringen Erfolgsbilanz sah die Europäische Kommission mit Ausbruch der Krise in der Stärkung dieser bestehenden Haushaltsbeschränkungen den vorrangigen Weg, die langfristige Stabilität der öffentlichen Haushalte in den Mitgliedstaaten sicherzustellen. Als eine frühe Reaktion auf die neuen Herausforderungen durch die Finanz- und Schuldenkrise wurde im Mai 2010 eine Arbeitsgruppe eingerichtet, die unter Vorsitz des Präsidenten des Europäischen Rates, Herman van Rompuy, Vorschläge für die wirtschaftspolitische Steuerung in Europa erarbeitete. Die im Oktober desselben Jahres vorgelegten Empfehlungen dienten als Grundlage für die Reformen des SWP, die schließlich im November 2011 in Form von fünf Verordnungen ${ }^{9}$ und einer Richtlinie ${ }^{10}$ (Sixpack) beschlossen wurden. Neben der neu eingeführten Überwachung der makroökonomischen Ungleichgewichte, auf die im Folgenden nicht weiter eingegangen wird, dienen diese Reformen hauptsächlich der Erweiterung und erleichterten Durchsetzbarkeit des korrektiven und präventiven Arms des SWP (siehe Tabelle 1).

Insbesondere sollte das Verfahren bei einem übermäßigen Defizit um Automatismen ergänzt werden, die sicherstellen, dass Sanktionen beim Überschreiten der weiterhin gültigen Verschuldungsgrenze von 3 Prozent des BIP tatsächlich umgesetzt werden. Der reformierte korrektive Arm führte hier nur zu einer partiellen Verschärfung, da die Abstimmungen im Rat der Europäischen Union über die neu eingeführten Sanktionsmöglichkeiten auf früheren Stufen ${ }^{11}$ nach dem Prinzip der ,umgekehrten qualifizierten Mehrheit' durchgeführt werden. Somit gilt dort ein Vorschlag der Europäischen Kommission als angenommen, wenn sich keine qualifizierte Mehrheit dagegen ausspricht. Dieses gilt jedoch nicht für frühere Verfahrensschritte, wie etwa bei der Feststellung eines übermäßigen Defizits, die ganz zu Beginn des Verfahrens auf Vorschlag der Europäischen Kommission erfolgen. Somit könnte eine Minderheit der Mitgliedstaaten weiterhin die Eröffnung des Defizitverfahrens verhindern. Dieses Manko sollte im Rahmen des Anfang 2012 vereinbarten Fiskalvertrags behoben wer-

8 Daniela Schwarzer: Zehn Jahre Governance der Eurozone: ökonomische Bilanz und institutionelle Dynamiken jenseits der Vertragsrevision, in: integration 1/2009, S. 17-32.

9 Verordnung (EU) Nr. 1173/2011 des Europäischen Parlaments und des Rates vom 16. November 2011 über die wirksame Durchsetzung der haushaltspolitischen Überwachung im Euro-Währungsgebiet; Verordnung (EU) Nr. 1174/2011 des Europäischen Parlaments und des Rates vom 16. November 2011 über Durchsetzungsmaßnahmen zur Korrektur übermäßiger makroökonomischer Ungleichgewichte im Euro-Währungsgebiet; Verordnung (EU) Nr. 1175/2011 des Europäischen Parlaments und des Rates vom 16. November 2011 zur Änderung der Verordnung (EG) Nr. 1466/97 des Rates über den Ausbau der haushaltspolitischen Überwachung und der Überwachung und Koordinierung der Wirtschaftspolitiken; Verordnung (EU) Nr. 1176/2011 des Europäischen Parlaments und des Rates vom 16. November 2011 über die Vermeidung und Korrektur makroökonomischer Ungleichgewichte; Verordnung (EU) Nr. 1177/2011 des Rates vom 8. November 2011 zur Änderung der Verordnung (EG) Nr. 1467/97 über die Beschleunigung und Klärung des Verfahrens bei einem übermäßigen Defizit.

10 Richtlinie 2011/85/EU über die Anforderungen an die haushaltspolitischen Rahmen der Mitgliedstaaten.

11 Siehe dazu später im Text. 
den. ${ }^{12}$ Da dieser jedoch nicht einvernehmlich beschlossen wurde und somit zunächst nicht im EU-Recht verankert werden konnte, war es nicht möglich, den SWP abermals zu ändern. Stattdessen wurde im Fiskalvertrag eine Verpflichtung vereinbart, die Empfehlungen der Europäischen Kommission im Rahmen eines Verfahrens bei einem übermäßigen Defizit zu unterstützen, wenn diese die Auffassung vertritt, dass ein Euro-Mitgliedstaat gegen das Defizit-Kriterium verstößt. Diese Entscheidung kann nur dann aufgehoben werden, wenn eine qualifizierte Mehrheit der Mitgliedstaaten dagegen votiert. Die Vereinbarung verfolgt somit das Ziel, mittels zwischenstaatlicher Vereinbarungen auch auf früheren Stufen des Verfahrens eine Abstimmung nach umgekehrter qualifizierter Mehrheit einzurichten.

Neben der Stärkung der Automatismen im Sanktionsverfahren wurden auch die Strafen an sich verschärft. Nun ist es bereits in früheren Verfahrensschritten möglich, auf Vorschlag der Europäischen Kommission Strafen in Form von unverzinslichen Einlagen oder Geldbußen (beides in Höhe von 0,2 Prozent des BIP) zu erheben. ${ }^{13}$ Auch auf der letzten Stufe, nach nicht erfolgten Sanierungsmaßnahmen des Staates, wurde das Strafmaß verschärft: Sollte hier vor der Reform ,in der Regel“ eine unverzinste Einlage als Strafe dienen, ist nun eine Geldbuße vorgesehen, deren Höhe 0,2 Prozent bis 0,5 Prozent des BIP betragen soll. Nicht durchsetzen konnte sich jedoch die Forderung nach weiteren nicht-monetären Strafen wie etwa einem Entzug von Stimmrechten sündiger Mitgliedstaaten.

Tabelle 1: Reform des Stabilitäts- und Wachstumspakts

\begin{tabular}{|c|c|c|c|c|}
\hline & $\begin{array}{l}\text { Reform Korrekti- } \\
\text { ver Arm SWP (1) }\end{array}$ & $\begin{array}{l}\text { Reform Korrekti- } \\
\text { ver Arm SWP (2) }\end{array}$ & $\begin{array}{l}\text { Reform Präventi- } \\
\text { ver Arm SWP (1) }\end{array}$ & $\begin{array}{l}\text { Ergänzung Prä- } \\
\text { ventiver Arm } \\
\text { SWP (2) }\end{array}$ \\
\hline Ratsbeschluss & \multicolumn{4}{|l|}{ 16. November 2011} \\
\hline Teilnehmer & \multicolumn{4}{|c|}{ EU-27 (Sanktionen betreffen nur die Euro-Staaten) } \\
\hline $\begin{array}{l}\text { Rechtliche } \\
\text { Verankerung }\end{array}$ & \multicolumn{4}{|c|}{ EU-Sekundärrecht (ein Teil der Verordnungen des Sixpacks) } \\
\hline Gesetzestexte & $\begin{array}{l}(\mathrm{EU}) 1177 / 2011 ; \\
(\mathrm{EU}) 1173 / 2011\end{array}$ & $\begin{array}{l}(\mathrm{EU}) 1177 / 2011 ; \\
(\mathrm{EU}) 1173 / 2011\end{array}$ & $\begin{array}{l}(E U) 1175 / 2011 ; \\
(E U) 1173 / 2011\end{array}$ & (EU)1175/2011 \\
\hline Art der Regel & Defizitregel & $\begin{array}{l}\text { Schuldenstands- } \\
\text { regel }\end{array}$ & Defizitregel & Ausgabenregel \\
\hline Grenzwert & 3 Prozent des BIP & $\begin{array}{l}60 \text { Prozent des BIP, } \\
\text { Verringerung des } \\
\text { Abstands zum } \\
\text { Grenzwert um jähr- } \\
\text { lich ein Zwanzigs- } \\
\text { tel }\end{array}$ & $\begin{array}{l}\text { „mittelfristiges } \\
\text { Haushaltsziel“: län- } \\
\text { derspezifisches Ziel } \\
\text { für strukturelles } \\
\text { Defizit (max. 1 Pro- } \\
\text { zent des BIP) }\end{array}$ & $\begin{array}{l}\text { Wachstumsrate der } \\
\text { Ausgaben unter- } \\
\text { halb des Potenzial- } \\
\text { Wachstums des BIP }\end{array}$ \\
\hline Sanktionen & $\begin{array}{l}\text { Vielfältige Sankti- } \\
\text { onsmöglichkeiten, } \\
\text { bis hin zu Geldbuße } \\
\text { von max. 0,5 Pro- } \\
\text { zent des BIP }\end{array}$ & $\begin{array}{l}\text { Vielfältige Sankti- } \\
\text { onsmöglichkeiten, } \\
\text { bis hin zu Geldbuße } \\
\text { von max. 0,5 Pro- } \\
\text { zent des BIP }\end{array}$ & $\begin{array}{l}\text { Verwarnung; max. } \\
\text { Strafe: verzinsliche } \\
\text { Einlage von } 0,2 \\
\text { Prozent des BIP }\end{array}$ & $\begin{array}{l}\text { Verwarnung; max. } \\
\text { Strafe: verzinsliche } \\
\text { Einlage von } 0,2 \\
\text { Prozent des BIP }\end{array}$ \\
\hline
\end{tabular}

Quelle: Eigene Darstellung.

12 Siehe dazu später im Text.

13 Für eine Übersicht siehe Matthias Kullas: Kann der reformierte Stabilitäts- und Wachstumspakt den Euro retten? Absichten, Einsichten, Übersichten, Centrum für Europäische Politik (CEP): cep Studie, Oktober 2011. 
Darüber hinaus ist durch die Sixpack-Reformen auch der Geltungsbereich des Sanktionsmechanismus erweitert worden. Zwar galt das Defizitverfahren seit seiner Einführung auch für eine Überschreitung des Schuldenstandskriteriums (60 Prozent des BIP), jedoch war dieser Richtwert in der Vergangenheit nicht operationalisiert. Im Sixpack wurde an dieser Stelle die Regel getroffen, dass ein übermäßiges Defizit vorliegt, wenn der Schuldenstand 60 Prozent des BIP überschreitet und der Abstand zum Zielwert nicht jedes Jahr um mindestens ein Zwanzigstel reduziert wird. In der Praxis bedeutet diese Regel für manche Staaten einen deutlich größeren Anpassungsbedarf als die 3-Prozent-Defizitregel. Beispielsweise müsste Italien bei einem Schuldenstand von etwa 120 Prozent des BIP im Jahr 2012 einen jährlichen nominalen Überschuss von 3 Prozent des BIP erwirtschaften.

Weitere Änderungen wurden am präventiven Arm des SWP vorgenommen. Unverändert blieb die Begrenzung des strukturellen Defizits auf ein vom Mitgliedstaat gesetztes mittelfristiges Haushaltsziel, das maximal 1 Prozent des BIP betragen darf, sowie die Festlegung eines Anpassungspfades zu dessen Erreichung.

Schließlich können nun auch Verletzungen des präventiven Arms mit Sanktionen belangt werden, wenn ein Mitgliedstaat ,erheblich“von dessen Vorgaben abweicht. Dies ist der Fall, wenn das strukturelle Defizit um mehr als 0,5 Prozent des BIP höher ausfällt als erlaubt oder in zwei aufeinanderfolgenden Jahren jeweils größer als 0,25 Prozent des BIP ist. Jedoch erfolgt die Entscheidung zur Aufnahme eines Verfahrens auf Grundlage einer Gesamtbewertung, die einen wesentlich größeren diskretionären Spielraum als beim korrektiven Arm zulässt. Zudem fallen die möglichen Sanktionen beim korrektiven Arm deutlich weicher aus und können außer einer Verwarnung maximal in Form einer verzinslichen Einlage in Höhe von 0,1 Prozent des BIP verhängt werden.

\section{Die Richtlinie zum nationalen haushaltspolitischen Rahmen}

Bei allen vorgenommenen Präzisierungen des SWP hatten die Reformen von Beginn an jedoch nur begrenzte Aussichten, einen wirklichen Schub an neuer Glaubwürdigkeit zu bringen. Der SWP bleibt in der Marktbewertung durch sein Versagen über die vergangenen zehn Jahre nachhaltig diskreditiert. Aus diesem Grund wurden die Bemühungen zur Reform des SWP rasch von dem Versuch begleitet, ebenfalls stärker auf die nationale Budgetebene in verbindlicher Weise Einfluss zu nehmen.

Eine erste Verpflichtung zur Einführung von Fiskalregeln auf nationaler Ebene findet sich bereits im September 2010 in den ersten Legislativvorschlägen für eine Richtlinie, die später in den ,Anforderungen an die haushaltspolitischen Rahmen der Mitgliedstaaten “ als Teil des Sixpacks verankert wurde. ${ }^{14}$

Diese Richtlinie macht Vorgaben zur Rechnungslegung und Statistik: Hier haben die Mitgliedstaaten die Haushaltsdaten für alle staatlichen Teilsektoren zeitnah und im Einklang mit den Regeln der europäischen Statistik öffentlich verfügbar zu machen. Für ihre Finanzplanung haben die Mitgliedstaaten realistische makroökonomische Prognosen heranzuziehen, die „mit den aktuellsten Prognosen der Kommission und gegebenenfalls mit den Prognosen anderer unabhängiger Einrichtungen zu vergleichen"15 sind. Zusätzlich sind Bandbreiten für alternative Szenarien zu berechnen. Ebenfalls müssen die der Finanzplanung zugrunde liegenden makroökonomischen Prognosen veröffentlicht und ex post bewertet werden. Von Bedeutung sind außerdem die Vorgaben zum mittelfristigen haushaltspolitischen

14 Richtlinie 2011/85/EU über die Anforderungen an die haushaltspolitischen Rahmen der Mitgliedstaaten.

15 Art. 4, ebenda. 
Rahmen: Der Finanzplanungshorizont muss mindestens drei Jahre vorsehen. Für diesen mehrjährigen Rahmen müssen Haushaltsziele ,in Bezug auf gesamtstaatliches Defizit, Schuldenstand und andere zusammenfassende Finanzindikatoren" 16 formuliert werden. Die jährlichen Haushaltsgesetze müssen mit diesen Plänen konsistent sein. Um Maßnahmen der ,Budgetkosmetik' einzudämmen, müssen die genannten Bestimmungen alle Teilsektoren des Staates abdecken. Sämtliche Transaktionen außerbudgetärer Fonds sind in den regulären Haushaltsprozess zu integrieren. Auch sind für alle Teilsektoren Informationen über Eventualverbindlichkeiten bereitzustellen.

Wesentlichster Inhalt der Richtlinie ist es aber, dass jeder Mitgliedstaat zur Einführung numerischer Haushaltsregeln, ,die wirksam zur Einhaltung ihrer jeweiligen aus dem AEUV im Bereich der Haushaltspolitik erwachsenden Verpflichtungen über einem Zeithorizont von mehreren Jahren durch den Staat als Ganzes beitragen“, ${ }^{17}$ verpflichtet wird. Dabei werden insbesondere Regeln genannt, die sich auf die vertraglich festgelegten Referenzwerte für Defizit und Schuldenstand beziehen. Die numerischen Regeln müssen Angaben zu ihren Zielvorgaben und Anwendungsbereichen, ihrer Überwachung, den Folgen bei Nichteinhaltung und Ausnahmetatbeständen beinhalten, die wirksam zur Einhaltung ihrer Verpflichtungen im Rahmen des SWP sind. ${ }^{18}$

Während diese Richtlinie somit bereits den Weg beschreitet, die Anpassung nationaler Budgetregeln voranzutreiben, bleibt sie bei ihren Vorgaben vage. Eine numerische Haushaltsregel wird zwar vorgeschrieben, aber nicht konkret spezifiziert. Außerdem werden keine Sanktionen angegeben, sodass bei Nichtumsetzung lediglich das langwierige allgemeine Vertragsverletzungsverfahren droht.

\section{Der Fiskalvertrag}

Den letzten weitgehendsten Vorstoß zur koordinierten Etablierung nationaler Fiskalregeln stellt schließlich der Fiskalvertrag dar, der im Dezember 2011 vereinbart und im März 2012 von den Staats- und Regierungschefs unterzeichnet wurde. Diese Vereinbarung sollte zunächst im Primärrecht der Europäischen Union verankert werden, was kurzfristig aufgrund des Widerstands einzelner Mitgliedstaaten nicht realisierbar war. Ersatzweise wurde nun von 25 EU-Mitgliedstaaten ${ }^{19}$ der Fiskalvertrag als völkerrechtlicher Vertrag ausgehandelt. Vorbehaltlich der Ratifizierung von bis dahin mindestens zwölf Euro-Staaten soll dieser zum 1. Januar 2013 in Kraft treten.

Der Fiskalvertrag bessert einige Schwächen des SWP nach, indem er beispielsweise die Geltung der umgekehrten qualifizierten Mehrheit in den SWP-Verfahren ausweitet. ${ }^{20}$ Seine wesentliche Innovation ist aber vor allem die verbindliche Einigung auf die koordinierte Einführung von detailliert spezifizierten Fiskalregeln auf nationaler Ebene.

Im Detail verpflichten sich die Staaten im Fiskalvertrag zu einem ausgeglichenen Haushalt, wobei maximal ein jährliches strukturelles Defizit von 0,5 Prozent des BIP zulässig ist. Für Staaten mit einer Verschuldung von deutlich unterhalb von 60 Prozent des BIP erhöht sich dieser Grenzwert auf ein maximal zulässiges strukturelles Defizit von 1 Prozent. Insgesamt liegt der Grenzwert in einer normalen konjunkturellen Lage somit unterhalb der ver-

16 Art. 9, ebenda.

17 Art. 5, ebenda.

18 Art. 6, ebenda.

19 Hierbei handelt es sich um die EU-27 mit Ausnahme des Vereinigten Königreichs und der Tschechischen Republik.

20 Siehe früher im Text. 
gleichbaren Grenzwerte für das Defizit, die im korrektiven Arm des SWP ein maximales Defizit von 3 Prozent und im präventiven Arm ein maximales mittelfristiges Haushaltsziel von 1 Prozent vorschreiben, wobei bereits heute alle Euro-Mitgliedstaaten ein mittelfristiges Haushaltsziel von 0,5 Prozent oder sogar besser verfolgen. ${ }^{21}$ Hinsichtlich der Neuverschuldung stellt die Vorgabe des Fiskalvertrages somit die restriktivste der neu beschlossenen Regelungen dar. Dies impliziert, dass eine Einhaltung der neuen nationalen Fiskalregeln auch die Einhaltung der Vorgaben des SWP sicherstellt. Eine Ausnahme ist das neue Schuldenstandskriterium des SWP. ${ }^{22}$ Im Falle eines sehr hohen Schuldenstands ist es somit möglich, dass trotz Einhaltung des Grenzwerts des Fiskalvertrages keine Rückführung des Schuldenstandes um ein Zwanzigstel des überschüssigen Betrages erfolgt.

Die Verankerung der Vorgaben des Fiskalvertrages im nationalen Recht der Mitgliedstaaten muss in Form von Bestimmungen erfolgen, ,die verbindlicher und dauerhafter Art sind, vorzugsweise mit Verfassungsrang, oder deren vollständige Einhaltung und Befolgung im gesamten nationalen Haushaltsverfahren auf andere Weise garantiert ist". ${ }^{23}$ Aufgrund unterschiedlicher Rechtstraditionen der Mitgliedstaaten musste an dieser Stelle jedoch von der ursprünglichen Forderung einer zwingenden Verfassungsverankerung Abstand genommen werden. Weitere Anforderungen an die einzuführenden Fiskalregeln bestehen in der Einrichtung unabhängiger nationaler Institutionen zur Überwachung der Einhaltung der Regeln sowie eines automatischen Korrekturmechanismus, der die Korrektur von Abweichungen in Bezug auf Art, Umfang und zeitlichen Rahmen regelt. Beides soll sich auf Grundsätze stützen, die noch von der Europäischen Kommission zu erarbeiten sind. Diese noch nicht bekannten Grundsätze regeln somit wichtige Details der nationalen Schuldenbremsen, die von großer Bedeutung für die Beurteilung ihrer Stringenz sind.

Eine Sanktionierungsmöglichkeit auf EU-Ebene bei Verstößen gegen die nationalen Schuldenbremsen ist im Fiskalvertrag nicht vorgesehen. Jedoch sind eine starke Überwachung der Einführung der nationalen Regelungen und deren Vereinbarkeit mit den genannten Vorgaben der Europäischen Kommission geplant. Im Detail wird die Einführung der Regeln durch einen Bericht der Europäischen Kommission mit Stellungnahme begleitet. Zudem hat jeder übrige am Fiskalpakt teilnehmende Staat die Möglichkeit, den Europäischen Gerichtshof (EuGH) mit der Angelegenheit zu befassen, wenn er der Meinung ist, dass ein anderer Staat seinen Verpflichtungen nicht nachgekommen ist. Ist dies der Fall, kann das Gericht in letzter Instanz eine Strafe von bis zu 0,1 Prozent des BIP verhängen. Dieses Verfahren mit Sanktionsmöglichkeit durch den EuGH ohne Mitwirkung des Rats der Europäischen Union verspricht somit eine deutlich stärkere Durchsetzungsfähigkeit als die oben angesprochenen Verfahren im Rahmen des SWP. Gemäß Fiskalvertrag entscheiden unabhängige Richter über die Verhängung der Sanktion, während im SWP verantwortlich handelnde Politiker selbst die Entscheidung treffen. Allerdings ist zu betonen, dass sich die fiskalvertragliche neue Sanktionsmöglichkeit nur auf die Vorschrift zur Etablierung einer nationalen Fiskalregel bezieht, nicht jedoch auf eine Verletzung dieser Regel. Für die Überwachung der Einhaltung der nationalen Regeln bleiben die nationalen Gerichte verantwortlich.

Tabelle 2 fasst die wesentlichen Unterschiede zwischen den Vorgaben und den neuen Fiskalregeln zusammen, die durch Sixpack, Euro-Plus-Pakt und den Fiskalvertrag definiert worden sind. Der Euro-Plus-Pakt ist einbezogen, weil auch er die Verpflichtung für die teil-

21 Vgl. hierzu Tabelle 1.

22 Siehe hierzu Tabelle 1, Reform Korrektiver Arm SWP (2).

23 Art. 3 Abs. 2 Fiskalvertrag. 
nehmenden Mitgliedstaaten enthält, „die im Stabilitäts- und Wachstumspakt enthaltenen Haushaltsvorschriften der EU in nationales Recht umzusetzen“. ${ }^{24}$ Dabei wurde jedoch auch für den Euro-Plus-Pakt die ursprüngliche Forderung nach Verankerung in der Verfassung fallengelassen. Stattdessen können die Mitgliedstaaten selbst das Rechtsinstrument wählen. Dieses muss lediglich ,verbindlicher und dauerhafter Art“ ${ }^{\star 25}$ sein, sodass dies neben der Verfassung auch in einem Rahmengesetz festgeschrieben werden kann. Zudem wird die genaue Ausgestaltung der Regel ebenfalls von den Mitgliedstaaten gewählt. Als Beispiele werden eine ,Schuldenbremse', eine mit dem Primärsaldo verknüpfte Regel und eine Ausgabenregel genannt. Schließlich erschöpft sich das Eingriffsrecht der EU-Ebene darin, dass die Europäische Kommission die Gelegenheit erhalten soll, zu der Haushaltsvorschrift angehört zu werden. Dies soll sicherstellen, dass die getroffene Vorschrift „,mit den EU-Vorschriften vereinbar ist und diese unterstützt“ ${ }^{26}$ Der Vergleich zeigt, dass der Fiskalvertrag eindeutig die am weitgehendsten und konkretesten Vorgaben für neue nationale Fiskalregeln mit sich bringt.

Tabelle 2: Regelungen zu nationalen Fiskalregelungen

\begin{tabular}{|c|c|c|c|}
\hline & Sixpack & Euro-Plus-Pakt & Fiskalvertrag \\
\hline Ratsbeschluss & 8. November 2011 & 25. März 2011 & 2. März 2012 \\
\hline Teilnehmer & EU-27 ohne UK & $\begin{array}{l}\text { Eurostaaten plus BG, } \\
\text { DK, LT, LV, PL, RO }\end{array}$ & EU-27 ohne CZ, UK \\
\hline $\begin{array}{l}\text { Rechtliche } \\
\text { Verankerung }\end{array}$ & $\begin{array}{l}\text { EU-Sekundärrecht } \\
\text { (Richtlinie 2011/85/EU) }\end{array}$ & $\begin{array}{l}\text { Vereinbarung zur frei- } \\
\text { willigen Selbstverpflich- } \\
\text { tung }\end{array}$ & Völkerrechtlicher Vertrag \\
\hline Grenzwert & Keine Angabe & Keine Angabe & $\begin{array}{l}\text { Strukturelles Defizit unter- } \\
\text { halb 0,5 Prozent des BIP } \\
\text { (Staaten mit Schuldenstand } \\
\text { unterhalb } 60 \text { Prozent: } 1 \\
\text { Prozent) }\end{array}$ \\
\hline Vorgaben & $\begin{array}{l}\text { Einführung länderspezi- } \\
\text { fischer numerischer } \\
\text { Haushaltsregeln zur Ein- } \\
\text { haltung der Referenzwer- } \\
\text { te des SWP }\end{array}$ & $\begin{array}{l}\text { Verpflichtung zur Um- } \\
\text { setzung der Haushalts- } \\
\text { vorschriften des SWP in } \\
\text { nationales Recht; freie } \\
\text { Wahl des Rechtsinstru- } \\
\text { ments (ausreichend ver- } \\
\text { bindlich und dauerhaft) }\end{array}$ & $\begin{array}{l}\text { Einführung von Bestim- } \\
\text { mungen im einzelstaatli- } \\
\text { chen Recht von ,,verbindli- } \\
\text { cher und dauerhafter Art“, } \\
\text { „vorzugsweise mit Verfas- } \\
\text { sungsrang“; Einrichtung ei- } \\
\text { nes automatischen Korrek- } \\
\text { turmechanismus auf der Ba- } \\
\text { sis eines Vorschlags der } \\
\text { Kommission }\end{array}$ \\
\hline $\begin{array}{l}\text { Eingriffsmöglich- } \\
\text { keiten der EU- } \\
\text { Ebene }\end{array}$ & Keine Angabe & $\begin{array}{l}\text { Anhörung der Kommis- } \\
\text { sion vor Erlass der Regel }\end{array}$ & $\begin{array}{l}\text { Bericht der Kommission; } \\
\text { Klagemöglichkeit der Mit- } \\
\text { gliedstaaten beim EuGH }\end{array}$ \\
\hline Sanktionen & Keine Angabe & Keine Angabe & $\begin{array}{l}\text { Strafe bei Nichteinführung } \\
\text { der Regel von bis zu 0,1 Pro- } \\
\text { zent des BIP }\end{array}$ \\
\hline
\end{tabular}

Quelle: Eigene Darstellung.

24 Europäischer Rat: Europäischer Rat 24./25. März 2011. Schlussfolgerungen, EUCO 10/1/11.

25 Art. 3 Abs. 2 Fiskalvertrag.

26 Europäischer Rat: Europäischer Rat 24./25. März 2011. Schlussfolgerungen, EUCO 10/1/11. 


\section{Empirie zur Wirksamkeit von Fiskalregeln}

\section{Historie und Typologie von Fiskalregeln}

Um Anhaltspunkte über die mögliche Wirksamkeit des Fiskalvertrags und der neuen verbindlichen nationalen Fiskalregeln zu gewinnen, kann auf eine inzwischen breite empirische Literatur zurückgegriffen werden, die sich im Wesentlichen auf die USA, die Europäische Union und die Schweiz bezieht. Daneben existieren noch einige wenige Studien, deren Datengrundlage die OECD-Staaten bilden.

Diese Analysen machen sich die lange Historie numerischer Fiskalregeln zunutze. Sogenannte ,goldene Regeln“ kamen bereits vereinzelt Mitte des 19. Jahrhunderts in den Vereinigten Staaten und etwas später in manchen Schweizer Kantonen zum Einsatz. ${ }^{27}$ Seit Mitte des 20. Jahrhunderts ist ihre Verbreitung schubweise gestiegen und durch eine anhaltend hohe Dynamik seit Beginn der 1990er Jahre gezeichnet. ${ }^{28}$

Im Rahmen dieser Literatur hat sich auch eine hilfreiche Typologie von Fiskalregeln herausgebildet. Ganz allgemein stellt eine Fiskalregel eine permanente Beschränkung der Fiskalpolitik durch einen numerischen Grenzwert dar, der sich auf verschiedene Fiskalindikatoren beziehen kann. ${ }^{29}$ Aufgegliedert nach der jeweiligen Zielkategorie lassen sich vier verschiedene Grundtypen von Fiskalregeln unterscheiden: ${ }^{30}$ (1) Saldoregeln haben den Budgetsaldo als Ziel. Unterschiede in der Ausgestaltung bestehen dabei hinsichtlich der genauen Anforderungen an den Budgetsaldo. Eine ,Balanced-Budget-Rule‘ etwa fordert den Budgetausgleich ein, andere Saldenregeln (SWP, deutsche Schuldenbremse für die Bundesebene und nun auch der Fiskalvertrag) geben eine verbindliche Obergrenze für das Defizit vor, die aber größer als null ist. (2) Schuldengrenzen setzen hingegen nicht an der Stromgröße des Budgetsaldos, sondern an der Bestandsgröße des Schuldenstandes an. Dabei wird die Staatsverschuldung absolut oder im Verhältnis zum BIP auf einen Zielwert begrenzt beziehungsweise die Entwicklung zu diesem Zielwert vorgeschrieben. Demgegenüber fokussieren (3) Ausgabenregeln auf die Entwicklung der öffentlichen Ausgaben (je nach Ausgestaltung als Gesamt- oder Primärausgaben) in absoluten Werten, im Verhältnis zum BIP oder in Wachstumsraten, wohingegen (4) Einnahmeregeln eine Unter- oder Obergrenze für die öffentlichen Einnahmen setzen. Hierbei kann beispielsweise die Verwendung von außergewöhnlichen Einnahmen (,windfall revenues ${ }^{\circ}$ ) vorgeschrieben werden. ${ }^{31}$

Im Hinblick auf den Einsatz der vier grundlegenden Typen numerischer Fiskalregeln lassen sich klare Entwicklungstendenzen festhalten: Während im Jahr 1990 hauptsächlich Saldoregeln und Schuldengrenzen eingesetzt wurden, hat die Anwendung von Einnahme- und insbesondere Ausgaberegeln in den Folgejahren deutlich zugenommen. Nichtsdestotrotz

27 George Kopits: Fiscal Rules: Useful Policy Framework or Unnecessary Ornament?, Internationaler Währungsfonds, Fiscal Affairs Department: IMF Working Paper 01/145, September 2001.

28 Internationaler Währungsfonds, Fiscal Affairs Department: Fiscal Rules - Anchoring Expectations for Sustainable Public Finances, Dezember 2009.

29 George Kopits/Steven Symansky: Fiscal Policy Rules, Internationaler Währungsfonds: IMF Occasional Paper 162, Washington 1998.

30 Europäische Kommission, Generaldirektion Wirtschaft und Finanzen: Public Finances in EMU 2006, European Economy 3/2006; Internationaler Währungsfonds: Fiscal Rules, 2009.

31 Für einen Überblick über diese und weitere Ausgestaltungsmöglichkeiten siehe Internationaler Währungsfonds: Fiscal Rules, 2009. 
bilden Saldo- und Schuldenregeln zusammen nach wie vor die Mehrheit der eingesetzten Regeln. ${ }^{32}$

Auch Europa hat vor Ausbruch der Schuldenkrise und der Einigung auf den Fiskalvertrag zunehmend Erfahrungen mit solchen Regeln sammeln können. So zählt die Europäische Kommission für das Jahr 2010 in den EU-Staaten bereits insgesamt 70 numerische Regeln. ${ }^{33}$ Während zu Beginn der 1990er Jahre Fiskalregeln vor allem auf der regionalen beziehungsweise lokalen Ebene implementiert waren, bezieht sich die Ausgestaltung am aktuellen Rand häufiger auf den Gesamtstaat sowie die zentrale Regierungsebene.

Eine wichtige Rolle für die Konzeption des Fiskalvertrags hat unter den jüngeren Regeln sicherlich die neue deutsche grundgesetzliche Schuldenbremse gespielt, die 2009 beschlossen wurde und sich stark am Vorbild der älteren schweizerischen Schuldenbremse orientiert. Die als Saldoregel konzipierte Vorschrift begrenzt das strukturelle Defizit der Bundesländer ab dem Jahr 2020 auf null Prozent und das des Bundes ab dem Jahr 2016 auf 0,35 Prozent des BIP. ${ }^{34}$

Ganz eindeutig hat die Finanz- und Schuldenkrise schon vor der Einigung auf den Fiskalvertrag den Prozess in Europa weiter beschleunigt: ${ }^{35}$ In Italien trat zum Januar 2010 ein Reformpaket in Kraft, welches den Fiskalföderalismus zwischen den verschiedenen staatlichen Ebenen neu regelt. Beispielsweise werden Haushaltsziele der mittelfristigen Finanzplanung nun für jede Ebene gesondert spezifiziert. Das im Vereinigten Königreich 2010 neu eingeführte fiskalische Regelwerk beinhaltet unter anderem eine Saldoregel, die an den Konjunkturzyklus angepasst ist. Dieser Vorgabe wird eine Schuldengrenze zur Seite gestellt, die eine Reduzierung des Schuldenstandes des öffentlichen Sektors bis 2015/2016 vorschreibt. Ebenfalls im Jahr 2010 wurde in Portugal ein neuer haushaltsrechtlicher Rahmen vereinbart. Neben der Etablierung einer mehrjährigen Finanzplanung mit Salden- und Ausgabenregeln soll ebenfalls ein unabhängiges Überwachungsorgan (,Fiscal Council`) eingesetzt werden. Darüber hinaus sind weitere Änderungen absehbar. In Irland zum Beispiel weisen Reformtendenzen hin zur Einführung einer Saldoregel, die den Primärsaldo auf 0,5 Prozent des BIP begrenzen soll. Eine zusätzlich eingeführte Ausgabenregel soll darüber hinaus ein höheres Ausgabenwachstum als die Wachstumsrate des BIP verhindern.

In jüngster Zeit wird in zahlreichen Staaten darüber hinaus die Einführung von Fiskalregeln diskutiert, die das strukturelle Defizit begrenzen sollen und somit offensichtlich dem Vorbild der deutschen Schuldenbremse folgen. Eine derartige Regelung wurde in den vergangenen Monaten bereits in Spanien und Österreich beschlossen. Die spanische Regelung (Begrenzung des strukturellen Defizits auf 0,4 Prozent des BIP) wurde in die Verfassung aufgenommen, wohingegen in Österreich (Begrenzung auf 0,35 Prozent des BIP) aufgrund des Widerstands der Opposition nicht die notwendige Mehrheit für eine Verfassungsänderung erreicht wurde und die Schuldenbremse lediglich im Bundeshaushaltsgesetz festgeschrieben wurde.

Es fällt somit auf, dass insbesondere einige der mit besonders hohen Schulden kämpfenden Staaten ganz autonom auf neue Fiskalregeln gesetzt haben, noch bevor sie der Fiskalvertrag dazu verpflichten konnte. Bemerkenswert ist hierbei, dass auch das Vereinigte Königreich

32 Europäische Kommission, Generaldirektion Wirtschaft und Finanzen: Public Finances in EMU 2010, European Economy 4/2010.

33 Europäische Kommission, Generaldirektion Wirtschaft und Finanzen: Fiscal Rules Database, 2010.

34 Art. 109 Abs. 3 i.V.m. Art. 115 Abs. 2 Grundgesetz.

35 Europäische Kommission, Generaldirektion Wirtschaft und Finanzen: Public Finances in EMU 2011, European Economy 3/2011. 
auf neue Regeln setzt, gleichzeitig aber europäische Vorgaben auf diesem Gebiet ablehnt. Im Folgenden wird die bisherige empirische Evidenz zur Wirksamkeit nationaler und subnationaler Fiskalregeln vorgestellt.

USA

Ende der 1970er Jahre wurden in zahlreichen Bundesstaaten der USA, Tax and Expenditure Limitations Laws‘ (Einnahme- und Ausgabenregeln, TEL) eingeführt. Diese Gesetze haben die Begrenzung staatlicher Aktivitäten und der Steuerbelastung zum Ziel, nicht jedoch die Schuldenbegrenzung. Diese Budgetregeln wurden immer wieder in ihren Wirkungen evaluiert. So findet Elder einen signifikant vermindernden Einfluss der Ausgabenbeschränkungen auf das Wachstum der Steuereinnahmen. ${ }^{36}$ Die Analyse von Shadbegian deutet auf einen signifikant vermindernden Einfluss der TEL-Gesetze sowohl auf die Ausgaben als auch auf die Steuereinnahmen hin. ${ }^{37}$ Auch in einer methodisch verfeinerten Analyse auf Basis eines Panels von US-Counties bestätigt Shadbegian diesen Befund. ${ }^{38}$

Neben den Einnahme- und Ausgabenregeln wurden in den USA auch die Saldo- und Schuldenregeln der Bundesstaaten auf ihre Wirksamkeit hin untersucht, mit teilweise widersprüchlichen Resultaten. Von Hagen betrachtet den Einfluss der gesetzlich festgelegten Verschuldungsgrenzen der US-Bundesstaaten auf die Verschuldung. Wie auch Kiewiet und Szakaly findet er Hinweise auf ,kreative Buchführung ${ }^{6}{ }^{39}$ Die Einführung von Fiskalregeln auf der Ebene der Bundesstaaten hat somit vor allem zu einer Verlagerung der Schulden auf die Gemeindeebene geführt.

Hingegen zeigen Eichengreen und Bayoumi in einer Panel-Analyse für die US-Bundesstaaten, dass Saldoregeln einen vermindernden Effekt auf die Defizite ausüben. ${ }^{40}$ Auch Bohn und Inman, ${ }^{41}$ Alt und Lowry ${ }^{42}$ sowie Alesina und Bayoumi ${ }^{43}$ können einen solchen Effekt nachweisen. Demnach gehen stringentere Saldoregeln mit geringeren Defiziten einher. Poterba zeigt außerdem, dass US-Bundesstaaten mit vergleichsweise stringenteren Defizitregeln ihre Ausgaben und Einnahmen infolge eines unerwarteten Defizitschocks schneller anpassen. $^{44}$

36 Harold W. Elder: Exploring the Tax Revolt: an Analysis of the Effects of State Tax and Expenditure Limitation Laws, in: Public Finance Review 1/1992, S. 47-63.

37 Ronald J. Shadbegian: Do Tax and Expenditure Limitations Affect the Size and Growth of State Government?, in: Contemporary Economic Policy 1/1996, S. 22-35.

38 Ronald J. Shadbegian: Do Tax and Expenditure Limitations Affect Local Government Budgets? Evidence From Panel Data, in: Public Finance Review 2/1998, S. 118-136; Ronald J. Shadbegian: Fiscal Federalism, Collusion, and Government Size: Evidence from the States, in: Public Finance Review 3/1999, S. 262-281.

39 Jürgen von Hagen: A Note on the Empirical Effectiveness of Formal Fiscal Restraints, in: Journal of Public Economics 2/1991, S. 191-210 und D. Roderick Kiewiet/Kristin Szakaly: Constitutional Limitations on Borrowing: An Analysis of State Bonded Indebtedness, in: Journal of Law, Economics and Organization 1/1996, S. 62-97.

40 Barry Eichengreen/Tamim Bayoumib: The Political Economy of Fiscal Restrictions: Implications for Europe from the United States, in: European Economic Review 3-4/1994, S. 783-791.

41 Henning Bohn/Robert P. Inman: Balanced Budget Rules and Public Deficits: Evidence from the U.S. States, National Bureau of Economic Research: NBER Working Paper Series No. 5533, 1996.

42 James E. Alt/Robert C. Lowry: Divided Government, Fiscal Institutions, and Budget Deficits: Evidence from the States, in: The American Political Science Review 4/1994, S. 811-828.

43 Alberto Alesina/Tamim Bayoumi: The Costs and Benefits of Fiscal Rules: Evidence from U.S. States, National Bureau of Economic Research: NBER Working Paper Series No. 5614, 1996.

44 James M. Poterba: State Responses to Fiscal Crises: The Effects of Budgetary Institutions and Politics, in: The Journal of Political Economy 4/1994, S. 799-821. 


\section{Europäische Union}

Eine der ersten Studien im europäischen Kontext wurde von der Europäischen Kommission durchgeführt. ${ }^{45}$ Darin wird analysiert, wie sich die Einführung der unterschiedlichen Fiskalregeln über den Zeitraum von 1990 bis 2005 in den EU-25-Staaten auf die konjunkturbereinigten Primärdefizite sowie auf die Höhe der Ausgaben ausgewirkt hat. Hierfür verwendet die Europäische Kommission ihren eigens dafür konstruierten Indikator (,FiscalRule-Index'). ${ }^{46}$ Dieser Index kombiniert quantitative (Anzahl der verschiedenen Regeln) sowie qualitative Merkmale der für einen Staat existierenden Fiskalregeln und verdichtet diese zu einer Kennzahl. Qualitative Merkmale betreffen zum Beispiel die gesetzliche Verankerung der Regel, die institutionellen Überwachungs- sowie Durchsetzungsmechanismen, implementierte automatische Sanktionen sowie die Öffentlichkeitswirksamkeit. ${ }^{47}$

Sowohl das konjunkturbereinigte Primärdefizit als auch die konjunkturbereinigten Primärausgaben der EU-Staaten werden dabei als abhängige Variable verwendet. Die Ergebnisse deuten auf einen signifikant vermindernden Einfluss stärkerer Fiskalregeln auf beide Größen hin. Demzufolge hat die vermehrte Einführung numerischer Regeln in zahlreichen EU-Staaten Anfang der 1990er Jahre maßgeblich zu einer Reduktion der Primärdefizite und -ausgaben beigetragen. Weitere Studien, deren Fokus ebenfalls auf dem Einfluss von Fiskalregeln in den europäischen Nationalstaaten liegt und deren Datengrundlage die zuvor genannte Studie ist, wurden von Debrun und Kumar ${ }^{48}$ sowie Debrun et al ${ }^{49}$ als auch Ayusoi-Casals et al. ${ }^{50}$ durchgeführt. Unter Berücksichtigung etwaiger Endogenitätsprobleme ${ }^{51}$ belegen auch diese Untersuchungen einen vermindernden Einfluss von Fiskalregeln auf die Primärdefizite.

Von Hagen und Wolff untersuchen explizit den Einfluss des SWP auf die Veränderungsraten der Schuldenstandsquoten in den EU-15-Staaten und bestätigen die Skepsis dem Pakt gegenüber: So hat der SWP offenbar vermehrt zu einer Ausweitung der kreativen Buchführung geführt. ${ }^{52}$

\section{OECD-Staaten}

In einer Studie für $24 \mathrm{OECD-Staaten} \mathrm{untersuchen} \mathrm{Guichard} \mathrm{et} \mathrm{al.,} \mathrm{wie} \mathrm{sich} \mathrm{die} \mathrm{Existenz}$ numerischer Fiskalregeln auf die Konsolidierungsanstrengungen, die Länge der Konsolidierungsperioden sowie auf die Stabilisierung der Schuldenstandsquoten der Länder ausge-

45 Europäische Kommission: Public Finances in EMU 2006, 2006.

46 Europäische Kommission: Fiscal Rules Database, 2010.

47 Für detaillierte Informationen zur Erstellung des Index, dessen Berechnungsmethode sowie eine kritische Bewertung siehe Europäische Kommission: Public Finances in EMU 2011, 2011.

48 Xavier Debrun/Manmohan S. Kumar: Fiscal Rules, Fiscal Councils and All That: Commitment Devices, Signaling Tools or Smokescreens?, in: Banca d'Italia (Hrsg.): Fiscal Policy: Current Issues and Challenges, Structural Economic Analysis Department. Public Finance Workshop, Rom 2007, S. 479-512.

49 Xavier Debrun/Laurent Moulin/Alessandro Turrini/Joaquim Ayuso-i-Casals/Manmohan S. Kumar: Tied to the Mast? National Fiscal Rules in the European Union, in: Economic Policy 54/2008, S. 297-362.

50 Joaquim Ayuso-i-Casals/Xavier Debrun/Manmohan S. Kumar/Laurent Moulin/Alessandro Turrini: Beyond the SGP - Features and Effects of EU National-Level Numerical Fiscal Rules, Konferenzvortrag: Economic Policy Panel, Lisbon 2007.

51 Das ,Endogenitätsproblem` erschwert den Beweis für eine klare Kausalität der Beziehung. So ist oftmals unklar, ob starke Fiskalregeln zu sinkenden Defiziten führen oder, ob umgekehrt, sich nur solche Staaten starke Fiskalregeln geben, die ohnehin stabilitätsorientiert sind und auch ohne Regeln niedrigere Defizite aufweisen würden. Vgl. dazu später die Ausführungen zu den schweizerischen Erfahrungen.

52 Jürgen von Hagen/Guntram B. Wolff: What Do Deficits Tell Us About Debt? Empirical Evidence on Creative Accounting with Fiscal Rules in the EU, in: Journal of Banking \& Finance 12/2006, S. 3259-3279. 
wirkt hat. ${ }^{53}$ Im Gegensatz zu den vorhergehenden Studien wird die Existenz einer numerischen Fiskalregel mithilfe einer Dummy-Variablen, die den Wert null/eins bei Nichtexistenz/ Existenz einer Regel annimmt, approximiert. Staaten mit einer Kombination aus Saldo- und Ausgabenregeln waren demnach erfolgreicher in der Konsolidierung ihrer Haushalte als Länder mit reinen Saldoregeln.

In einer weiteren Studie untersuchen Dahan und Strawczynski die Wirksamkeit von Saldound Ausgabenregeln im Hinblick auf die jeweiligen fiskalischen Größen. ${ }^{54}$ Mithilfe von Dummy-Variablen und Daten von 22 OECD-Ländern gelangen sie zu ähnlichen Ergebnissen wie Guichard et al. Darüber hinaus zeigen die Ergebnisse, dass der verringernde Effekt von Fiskalregeln auf die Defizite und Ausgaben mittelfristig mit einem Rückgang der Sozialausgaben (gemessen an den Gesamtausgaben) einhergeht.

\section{Schweiz}

Im subnationalen Kontext der Schweiz untersuchen Feld und Kirchgässner sowie Schaltegger wie sich die Einführung von numerischen Fiskalregeln sowohl auf kantonaler (26 Kantone) als auch auf lokaler Ebene (132 Städte/Gemeinden) auf die Entwicklung fiskalischer Größen (Ausgaben, Einnahmen, Defizite sowie Schulden) ausgewirkt hat. ${ }^{55}$ Die Autoren finden auf beiden föderalen Ebenen einen signifikant verringernden Einfluss stringenter Fiskalregeln auf die Defizite. Bezüglich der Verschuldung ergeben die Ergebnisse ebenfalls einen signifikant verringernden Einfluss auf kantonaler Ebene, wohingegen der Einfluss auf Einnahmen und Ausgaben insignifikant ist.

In einer weiteren Studie widmen sich Krogstrup und Wälti dem Endogenitätsproblem: Es ist vorstellbar, dass in bestimmten Gebietskörperschaften vermehrt Fiskalregeln eingeführt werden, weil die Bürger dieser Gebietskörperschaften stärkere Präferenzen für eine nachhaltige Fiskalpolitik aufweisen und die Politiker diesen Präferenzen Folge leisten. Ist dies der Fall, liegt eine Verzerrung aufgrund von ausgelassenen Variablen (,omitted variable bias $^{6}$ ) vor. Unter Einbezug eines von Funk und Gathmann ${ }^{56}$ konstruierten Maßes für die Wählerpräferenzen in den Kantonen untersuchen die Autoren erneut den Einfluss von Fiskalregeln auf die Höhe der Defizite. Die Ergebnisse zeigen, dass ein signifikant verringernder Einfluss von Fiskalregeln auch unter Einbezug der Wählerpräferenzen bestehen bleibt, der gegenüber den Ausgangsschätzungen jedoch etwas geringer ausfällt. ${ }^{57}$

\section{Ausgestaltung von Fiskalregeln als wesentliche Determinante der Wirksamkeit}

Insgesamt zeigt sich somit, dass nationale und sub-nationale numerische Fiskalregeln im Allgemeinen einen verringernden Einfluss auf fiskalische Größen wie Defizite oder Schul-

53 Stéphanie Guichard/Mike Kennedy/Eckhard Wurzel/Christophe André: What Promotes Fiscal Consolidation, OECD Economics Department: Working Papers No. 553, 2007.

54 Momi Dahan/Michel Strawczynski: Fiscal Rules and Composition Bias in OECD Countries, CESifo Working Paper No. 3088, München 2010.

55 Lars P. Feld/Gebhard Kirchgässner: The Political Economy of Direct Legislation: Direct Democracy and Local Decision-Making, in: Economic Policy 33/2001, S. 329-367 und Christoph A. Schaltegger: Budgetregeln und ihre Wirkung auf die öffentlichen Haushalte: Empirische Ergebnisse aus den US-Bundesstaaten und den Schweizer Kantonen, in: Schmollers Jahrbuch. Zeitschrift für Wirtschafts- und Sozialwissenschaften 3/2002, S. 369-413. In einer weiteren Studie kommen die Autoren zu ähnlichen Ergebnissen: Lars P. Feld/Gebhard Kirchgässner: On the Effectiveness of Debt Brakes: The Swiss Experience in Neck und Sturm, Sustainability of Public Debt, Cambridge/London 2008, S. 223-255.

56 Patricia Funk/Christina Gathmann: Estimating the Effect of Direct Democracy on Policy Outcomes: Preferences Matter!, Stanford Center for International Development: Working Paper No. 248, August 2005.

57 Signe Krogstrup/Sébastien Wälti: Do Fiscal Rules Cause Budgetary Outcomes?, in: Public Choice 1-2/2008, S. 123-138. 
den haben. Zwar ergibt eine Reihe älterer Studien keine signifikanten Effekte, was jedoch überwiegend auf unzureichend spezifizierte Schätzgleichungen oder auf die der Analyse zugrunde liegenden Untersuchungsansätze zurückzuführen ist. Eine weitere zentrale Einsicht ist, dass der dämpfende Effekt der Fiskalregeln umso höher ausfällt, je stringenter die Regeln ausgestaltet sind. So macht es beispielsweise durchaus einen Unterschied, ob eine Fiskalregel durch einfache Gesetzesänderungen oder nur durch eine Verfassungsänderung modifiziert werden kann. Aussagen darüber, inwiefern die Art der Fiskalregel (zum Beispiel Ausgaben- oder Einnahmeregel) auf die Höhe des Gesamteffekts ausschlaggebend ist, lassen sich aus den Studien jedoch nicht treffen, da bei den Schätzungen in der Regel Indikatoren herangezogen werden, die alle Arten von Fiskalregeln zu einem Indikator aggregieren.

Eine bedeutende Rolle bei der Einführung von numerischen Fiskalregeln spielt schließlich auch deren Ausgestaltung. Der Literaturüberblick legt dar, dass schlecht ausgestaltete Fiskalregeln zwar zu einer Reduktion von fiskalischen Größen führen können, im Gegenzug dafür aber die kreative Buchführung ausgeweitet wird. Gut ausgestaltete (numerische) Fiskalregeln sollten dies berücksichtigen und somit Ausweichmöglichkeiten jeglicher Art vermeiden.

\section{Bedeutung des Fiskalvertrags als Teilbeitrag einer umfassenden Gesamtstrategie}

Betrachtet man nun die europäischen Innovationen im fiskalischen Regelwerk im Licht dieser empirischen Erkenntnisse, dann ergibt sich ein vorsichtig optimistisches Bild. So ist es eine gut begründete Innovation, nicht mehr alleine auf europarechtlich definierte Fiskalregeln zu setzen, sondern das nationale Budgetrecht stärker in den Dienst zu nehmen. Der Misserfolg des SWP, die nationale Haushaltspolitik tatsächlich nachhaltiger zu gestalten, ist unter anderem darauf zurückzuführen, dass nationale Haushaltssysteme den Mitgliedstaaten nicht die notwendige budgetäre Steuerungsfähigkeit eröffnet haben. Der SWP ist dabei in seiner Glaubwürdigkeit durch die zurückliegenden schlechten Erfahrungen beschädigt, sodass seine oben beschriebene Reform richtig, aber alleine zu wenig wäre, um den Kapitalmärkten eine dauerhafte Wende in der Verschuldungspolitik zu signalisieren. Der Versuch, hier nun durch eine stärkere Beeinflussung nationaler Regeln einzugreifen, ist von daher gut begründet.

Schon die neue Richtlinie zum haushaltspolitischen Rahmen leistet dabei einen ersten Beitrag für mehr Glaubwürdigkeit. Anzuerkennen ist, dass sie mit ihren Vorschriften sehr breit ansetzt und helfen soll, die Vorbedingungen für wirksame Fiskalregeln zu verbessern. Denn solche Regeln können nur dann effektiv sein und an den Kapitalmärkten Vertrauen schaffen, wenn sie in einem Umfeld zuverlässiger statistischer Informationen und mehrjähriger Finanzplanungsprozesse implementiert werden.

Der durch den Fiskalvertrag vorgeschriebene Standard für künftige nationale Regeln stellt sich in Relation zu den bisher existierenden nationalen fiskalischen Regeln als durchaus zufriedenstellend dar und lässt von daher eine effektive Defizitbegrenzung erwarten.

Eine strenge Regel - etwa gemäß dem in der Empirie verwendeten Fiscal-Rule-Index der Europäischen Kommission - ist dadurch gekennzeichnet, dass sie sich umfassend auf die gesamtstaatliche Verschuldung bezieht, sodass die notorischen Versuche zur Bilanzkosmetik ins Leere laufen. Außerdem sollte sie möglichst von einer unabhängigen Institution überwacht werden, sodass Sanktionsdrohungen glaubwürdig sind. Zusätzlich sind eine große Öffentlichkeitswirkung von Vorteil und eine Absicherung im Verfassungsrecht wünschenswert. 
Der fiskalvertragliche Mindeststandard erfüllt durchaus einige dieser Bedingungen einer strengen und damit vermutlich wirksamen Regel. So bezieht sich die Vorschrift eindeutig auf das gesamtstaatliche Defizit. Damit ist der neue europäische Standard sogar strenger als die deutsche grundgesetzliche Schuldenbremse, weil die Sozialversicherungssysteme dort außen vor bleiben. Die umfassende Definition erschwert Umgehungsbemühungen. Durch die beim EuGH angesiedelte Sanktionsentscheidung ist eine hohe Glaubwürdigkeit der Sanktionsdrohung gegeben, die sich stark vorteilhaft von der politischen Einflussnahme auf SWP-Sanktionen unterscheidet. Allerdings bezieht sich die Fiskalvertrags-Sanktion nur auf die Einführung der Regel, nicht aber auf deren Einhaltung. Ein weiteres Manko des Fiskalvertrags ist die nicht gelungene zwingende verfassungsrechtliche Absicherung der nationalen Budgetregeln. Immerhin jedoch sichert der völkerrechtlich bindende Fiskalvertrag die Regel ein Stück weit gegen einfachgesetzliches Außerkraftsetzen ab. Ein Staat würde vertragsbrüchig, wenn er die nationale Regel wieder außer Kraft setzt. Damit ist die Disposition des nationalen Gesetzgebers über die neue Regel eingeschränkt, allerdings auch nicht vollständig eliminiert.

Eine weitere Einschränkung in der Bewertung ist, dass der Fiskalvertrag wichtige Details der neuen Fiskalregeln mit seiner Unterzeichnung noch offen gelassen hat. Dies gilt für die vorgeschriebene Geschwindigkeit in der Annäherung an den Zielwert, für die Ausgestaltung des Korrekturmechanismus, der die Verpflichtungen für den Fall regelt, dass es zu einer Abweichung der Budgetsalden vom Zielwert kommt, aber auch für die Vorgaben für die zur Überwachung der Regel zuständigen nationalen Institution. Hier ist die Europäische Kommission mit der Konkretisierung betraut (Art. 3 Abs. 1 und Art. 3 Abs. 2 Fiskalvertrag). Von dieser Konkretisierung wird es abhängen, ob der durch den Fiskalvertrag gesetzte Regelstandard noch verbessert werden kann. Hier ist es wünschenswert, dass ein solcher Korrekturmechanismus unbedingt ein institutionelles Gedächtnis für Zielverfehlungen erhält. Die Regeln müssen dafür sorgen, dass Abweichungen des Defizits nach oben innerhalb einer klar definierten kurzen Zeitspanne durch Unterschreitungen kompensiert werden. Nur so werden die Schuldenquoten effektiv zu stabilisieren sein. Auch müssen Ausnahmeklauseln im Rahmen eines solchen Korrekturmechanismus auf ein Minimum beschränkt bleiben.

Abschließend ist im Hinblick auf diese vorsichtig optimistische Bewertung vor der naiven Erwartung zu warnen, dass ein neuer Vertrag oder ein neues nationales Gesetz in Isolation in der Lage sein könnten, tief sitzende Verschuldungsursachen auszugleichen. Solche Regeln können überhaupt nur wirken, wenn gleichzeitig auch umfassende institutionelle Reformen von Staat und Wirtschaft eingeleitet werden. Insofern ist es überzogen, alleine im Hinblick auf den Fiskalvertrag von einer fiskalischen Zeitenwende zu sprechen. Immerhin aber deutet die Empirie darauf hin, dass nationale Fiskalregeln, wie sie nun durch den Fiskalvertrag vereinbart werden sollen, als Element einer umfassenden Gesamtstrategie tatsächlich einen nicht unwichtigen Teilbeitrag zur Vertrauensbildung leisten können. 